\title{
Epithelial-to-mesenchymal transition confers pericyte properties on cancer cells
}

\author{
Anitha K. Shenoy, ${ }^{1,2}$ Yue Jin, ${ }^{1}$ Huacheng Luo, ${ }^{1}$ Ming Tang,,${ }^{1}$ Christine Pampo, ${ }^{3}$ Rong Shao,,${ }^{4}$ Dietmar W. Siemann, ${ }^{3}$ Lizi Wu, ${ }^{5}$ \\ Coy D. Heldermon, ${ }^{6}$ Brian K. Law, ${ }^{7}$ Lung-Ji Chang, ${ }^{5}$ and Jianrong Lu ${ }^{1}$ \\ 'Department of Biochemistry and Molecular Biology, UF Health Cancer Center, University of Florida College of Medicine, Gainesville, Florida, USA. ²Department of Pharmaceutical and Biomedical Sciences, \\ California Health Sciences University, Clovis, California, USA. ${ }^{3}$ Department of Radiation Oncology, UF Health Cancer Center, University of Florida College of Medicine, Gainesville, Florida, USA. \\ ${ }^{4}$ Morrill Science Center, Department of Biology, University of Massachusetts, Amherst, Massachusetts, USA. ${ }^{5}$ Department of Molecular Genetics and Microbiology, ${ }^{6}$ Department of Medicine, and ${ }^{7}$ Department \\ of Pharmacology and Therapeutics, UF Health Cancer Center, University of Florida College of Medicine, Gainesville, Florida, USA.
}

\begin{abstract}
Carcinoma cells can acquire increased motility and invasiveness through epithelial-to-mesenchymal transition (EMT). However, the significance of EMT in cancer metastasis has been controversial, and the exact fates and functions of EMT cancer cells in vivo remain inadequately understood. Here, we tracked epithelial cancer cells that underwent inducible or spontaneous EMT in various tumor transplantation models. Unlike epithelial cells, the majority of EMT cancer cells were specifically located in the perivascular space and closely associated with blood vessels. EMT markedly activated multiple pericyte markers in carcinoma cells, in particular PDCFR- $\beta$ and $\mathrm{N}$-cadherin, which enabled EMT cells to be chemoattracted towards and physically interact with endothelium. In tumor xenografts generated from carcinoma cells that were prone to spontaneous EMT, a substantial fraction of the pericytes associated with tumor vasculature were derived from EMT cancer cells. Depletion of such EMT cells in transplanted tumors diminished pericyte coverage, impaired vascular integrity, and attenuated tumor growth. These findings suggest that EMT confers key pericyte attributes on cancer cells. The resulting EMT cells phenotypically and functionally resemble pericytes and are indispensable for vascular stabilization and sustained tumor growth. This study thus proposes a previously unrecognized role for EMT in cancer.
\end{abstract}

\section{Introduction}

The majority of human cancers arise in epithelial tissues. A defining feature of epithelial cells is that they can establish strong intercellular adhesion, which constrains cell mobility (1). However, epithelial cells are able to shed their epithelial characteristics via epithelial-to-mesenchymal transition (EMT), a reprogramming process first recognized in developmental studies in the $1980 \mathrm{~s}$ (2). In response to EMT-inducing signals, epithelial cells weaken or lose cell-cell adhesion, repress the expression of epithelial cell markers (including adhesion molecules such as E-cadherin), and activate mesenchymal genes (e.g., N-cadherin) (3). These changes endow cells of epithelial origin with the increased migratory and invasive capacity of mesenchymal cells.

Carcinoma cells undergoing spontaneous EMT have been identified in transgenic mouse mammary tumors and in human breast cancer specimens (4-6). As EMT may enable carcinoma cells to overcome cell-cell adhesion and to invade neighboring tissue, EMT has been proposed to be a critical event initiating tumor invasion and metastasis (7). According to the prevailing hypothesis, a small subset of carcinoma cells that are in close contact with surrounding stroma may receive EMT-inducing signals from the microenvironment, undergo EMT, and form the invasive front, thus setting the stage for metastatic dissemination $(3,7,8)$. Consistent with this theory, disruption of E-cadherin-mediated cell adhesion causes tumor

Conflict of interest: The authors have declared that no conflict of interest exists. Submitted: January 19, 2016; Accepted: September 1, 2016.

Reference information: J Clin Invest. 2016;126(11):4174-4186. doi:10.1172/JCI86623. invasion and metastasis in a transgenic mouse model of pancreatic $\beta$-cell cancer (9). Moreover, temporal transgenic expression of the EMT-inducing transcription factor Twist1 promotes metastasis in a mouse model of chemically induced skin carcinogenesis (10). These studies suggest that experimental induction of EMT may stimulate tumor metastatic progression in vivo.

However, the relevance of EMT in cancer metastasis has been controversial $(11,12)$. Ectopic induction of EMT fails to induce evident metastasis in transplantation and transgenic mouse tumor models $(13,14)$. Acquisition of mesenchymal traits by carcinoma cells may not facilitate metastasis (11). In human breast cancer, EMT does not predict metastasis and poor clinical outcome $(12,15)$. Importantly, recent cell lineage-tracing and genetic studies showed that EMT is dispensable for spontaneous metastasis in multiple transgenic mouse models of breast and pancreatic cancer (16-18). These findings suggest that EMT cancer cells may not be solely devoted to metastasis as previously suggested, and warrant a re-evaluation of the significance of EMT in cancer. Although EMT generates mesenchymal-like cells, the exact fates and roles of epithelial tumor cells naturally transitioning to a mesenchymal state in vivo remain largely unclear.

In the present study, we tracked carcinoma cells that underwent inducible or spontaneous EMT in various tumor transplantation models. The majority of EMT cancer cells are not enriched at the edge of tumors, but rather specifically located in perivascular space and closely associated with blood vessels, thereby simulating pericytes. Indeed, EMT cells express multiple pericyte markers and display gene expression patterns similar to those of pericytes. EMT enables cancer cells to attach to vascular endothelial cells and per- 


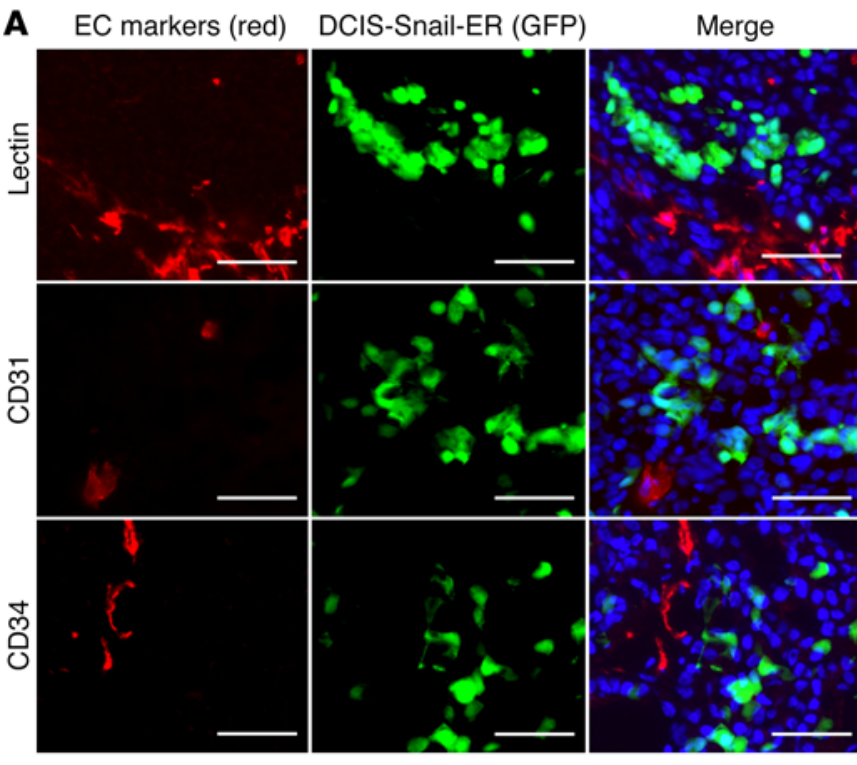

Vehicle (seasame oil)

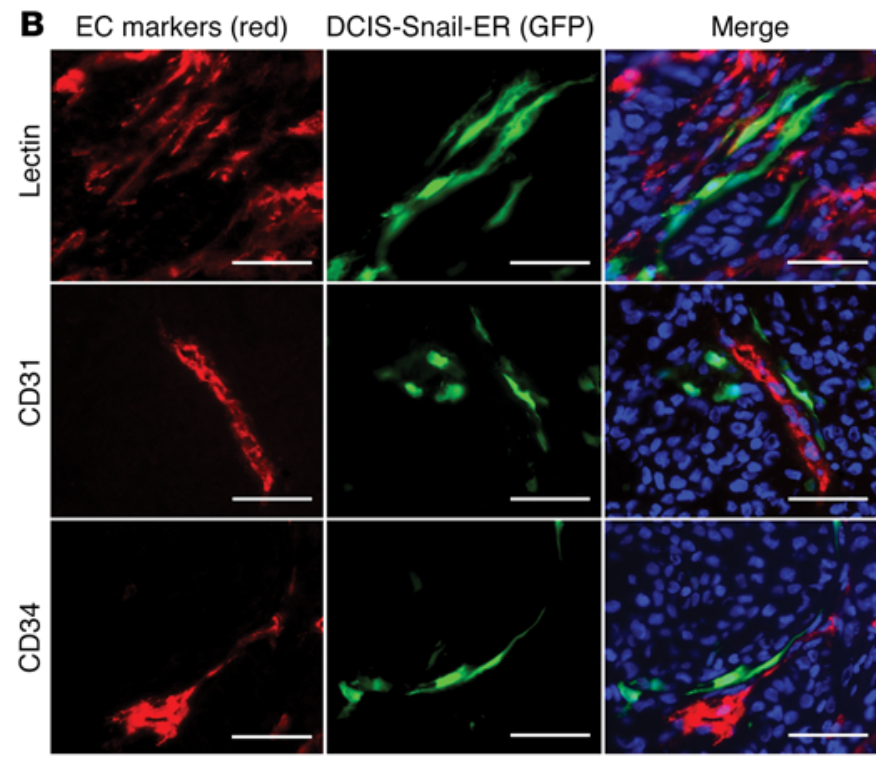

Tamoxifen

Figure 1. Induction of EMT enables cancer cells to associate with blood vessels. GFP-tagged DCIS-Snail-ER cells (green) were mixed with unlabeled DCIS epithelial cells (1:4) for orthotopic transplantation into immunodeficient mice. Tumor-bearing mice were treated with vehicle (A) or tamoxifen to induce EMT in DCIS-Snail-ER cells (B). Tumor vasculature was stained red with Griffonia simplicifolia lectin or antibodies against the endothelial markers CD31 (also known as PECAM1) and CD34. DNA was stained with Hoechst 33342 (blue). Scale bars: $50 \mu \mathrm{m}$.

form pericyte functions. Depletion of EMT cells abolishes pericyte coverage, leading to hyperpermeable vasculature and diminished tumor growth. The results suggest that EMT reprograms carcinoma cells into pericyte-like cells that are essential for tumor vascular stabilization, thus revealing a new promalignant effect of EMT.

\section{Results}

Mammary carcinoma cells that undergo EMT exhibit perivascular localization and close association with endothelium in vivo. To monitor EMT cells in vivo, we first established epithelial cells that could be induced to undergo EMT. The zinc finger transcription factor Snail is a central inducer of EMT $(19,20)$. Human mammary epithelial cells expressing an inducible form of Snail (Snail-ER) initiated EMT when treated with 4-hydroxytamoxifen (4HT) (21). We thus transduced MCF10DCIS transformed human mammary epithelial cells (referred to as DCIS) (22) with lentivirus expressing Snail-ER and GFP. The resultant GFP-tagged DCIS Snail-ER cells (referred to here as DCIS-Snail-ER) maintained an epithelial phenotype; however, they underwent characteristic EMT following 4HT treatment (Supplemental Figure 1A; supplemental material available online with this article; doi:10.1172/JCI86623DS1). As EMT usually occurs only in a minor fraction of carcinoma cells in the primary tumor, we sought to mimic the scenario by mixing the EMT-inducible DCIS-Snail-ER cells with parental DCIS epithelial cells at a 1:4 ratio, and injecting the cell mixture into the mouse mammary fat pad. The resulting tumors consisted of mostly unlabeled DCIS cells and a much smaller number of DCIS-Snail-ER cells that could be distinguished owing to their expression of GFP. Tumor-bearing mice were randomly divided into 2 groups, and received either vehicle (sesame oil) or tamoxifen to induce EMT of the DCIS-Snail-ER cells in tumor mass. In vehicle-treated tumors,
DCIS-Snail-ER cells showed a cuboidal-like epithelial morphology and a clonal growth pattern (Figure 1A). By contrast, in tamoxifen-treated tumors, most DCIS-Snail-ER cells became elongated, and interestingly, were aligned to display a capillary-like pattern that was reminiscent of blood vessels (Figure 1B). Therefore, we stained the blood vasculature in these tumors with lectin or antibodies against vascular endothelial cell (EC) markers CD31 and CD34 (Supplemental Figure 1B) (23). DCIS-Snail-ER cells were not stained by these endothelial markers (Figure 1B), suggesting that they are not converted into ECs. Instead, the majority of DCIS-Snail-ER cells exhibited periendothelial localization and were aligned with vasculature (Figure 1B and Supplemental Figure 1C). By contrast, DCIS-Snail-ER cells in control tumors were isolated from endothelial vasculature and did not show vascular alignment (Figure 1A). These observations suggest that EMT enables carcinoma cells to associate with vascular endothelium.

Mesenchymal cancer cells associate with vasculature in tumor xenograft. To determine whether the vascular-association property might be a general trait of EMT cells, we investigated multiple E-cadherin ${ }^{\mathrm{lo}} / \mathrm{N}$-cadherin ${ }^{\text {hi }}$ mesenchymal cancer cell lines (Supplemental Figure 2A), which featured a permanent EMT phenotype. Mesenchymal 786-O human renal carcinoma cells were stably labeled with GFP through lentiviral transduction, and subsequently mixed at a 1:4 ratio with unlabeled DCIS epithelial cells to generate tumor xenografts. Tumor vasculature was stained with lectin and antibodies recognizing the EC markers CD31 (also known as PECAM1), CD34, VE-cadherin, and VEGFR2 (also known as FLK1) (23). More than $70 \%$ of $786-O$ cells in these tumors were closely associated with ECs and in line with blood vasculature (Figure 2, A and B, and Supplemental Figure 2B). In a similar setting, when mixed with HCT116 colon or A549 lung epithelial cancer cells to 
A EC marker (red)
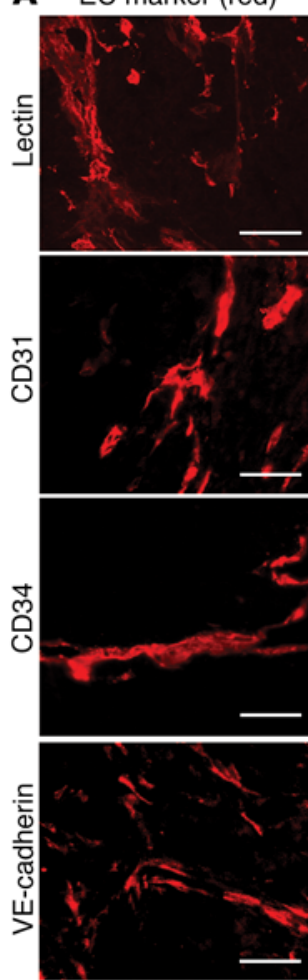

786-O (GFP)
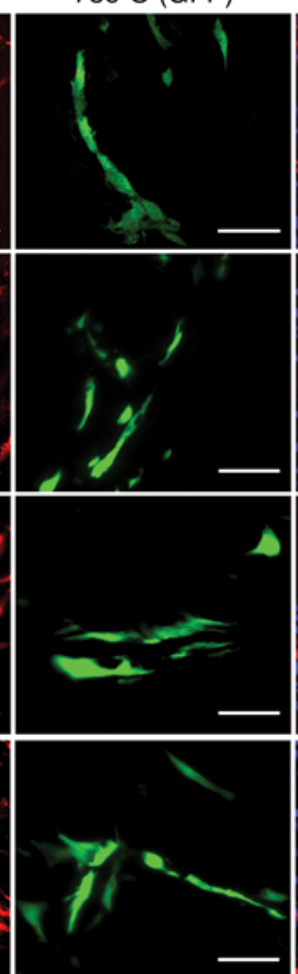

Merge
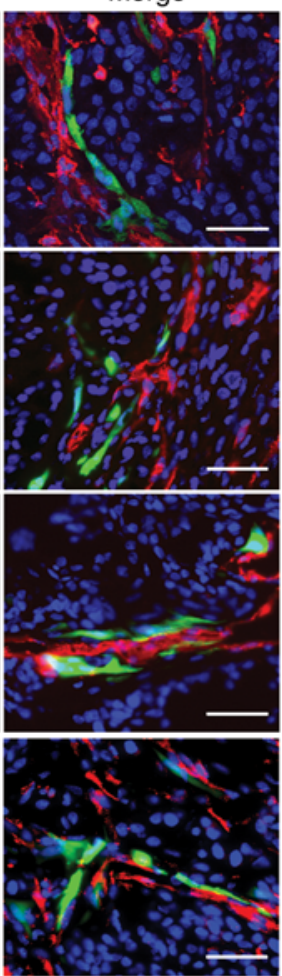

B

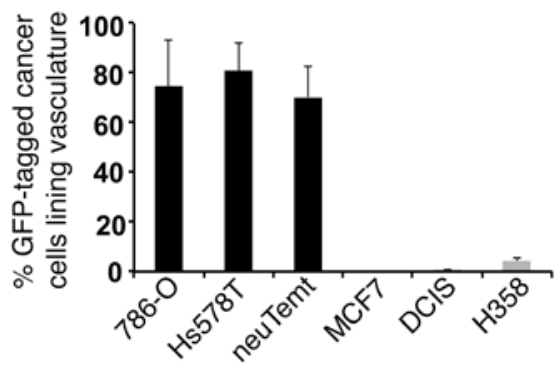

C

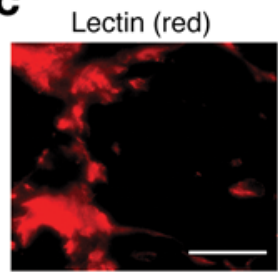

Lectin (red)

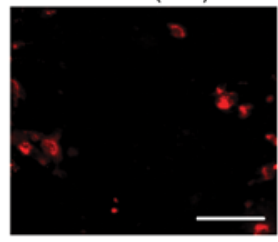

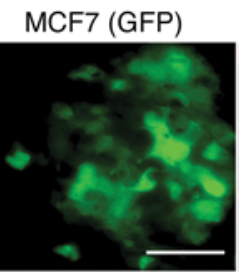

H358 (GFP)

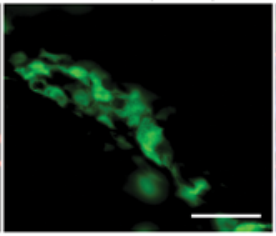

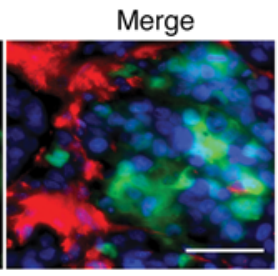

Merge

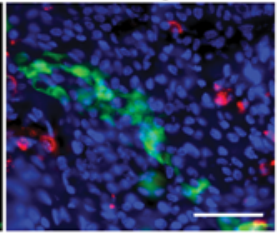

Figure 2. Mesenchymal cancer cells are associated with endothelial vasculature in tumor xenografts. (A) GFP-tagged mesenchymal 786-0 cells (green) were mixed with unlabeled epithelial DCIS cells (1:4) for tumor transplantation. Endothelial vasculature (red) in tumor sections was immunostained with Griffonia simplicifolia lectin or antibodies against endothelial markers CD31, CD34, and VE-cadherin. DNA was stained blue with Hoechst 33342. (B) Quantitative evaluation of various GFP-tagged mesenchymal and epithelial cancer cells that were associated with blood vessels in tumor xenografts resulting from the mixture of these cells with unlabeled DCIS cells. Error bars represent SD ( $n=3-4$ for each cell line). (C) GFP-tagged MCF7 or H358 epithelial cells (green) were mixed with unlabeled DCIS epithelial cells (1:4) to generate tumor xenografts. Tumor blood vessels were marked by G. simplicifolia lectin (red). DNA was stained blue. Scale bars: $50 \mu \mathrm{m}$.

generate tumor xenografts, GFP-marked 786-O cells also displayed close vascular association in tumors (Supplemental Figure 3A).

Mesenchymal neuTemt cells were derived from neuT mouse mammary epithelial tumor cells following EMT in vivo (24). Hs578T human triple-negative breast cancer cells were also mesenchymal. When neuTemt or Hs578T cells were stably tagged with GFP and mixed with unlabeled DCIS epithelial cells at a 1:4 ratio for orthotopic tumor transplantation, the majority of mesenchymal cancer cells closely aligned with vasculature as well (Figure 2B and Supplemental Figure 3B). By contrast, when GFP-tagged human epithelial cancer cells such as MCF7 breast cancer cells or H358 lung cancer cells were mixed with unlabeled DCIS cells to generate tumor xenografts, they formed clumps and were segregated from blood vessels (Figure 2, B and C). These results from various heterologous combinations of cancer cells suggest that mesenchymal cancer cells, but not epithelial cancer cells, are able to associate with blood vasculature in tumor xenografts.

However, in tumor xenografts generated purely from mesenchymal neuTemt cells, only a subset of these cells exhibited vascular association (Supplemental Figure 4). Similarly, in tumor xenografts derived entirely from DCIS-Snail-ER cells (without the mixture of parental DCIS cells), following the induction of EMT, only a minor population of cells was aligned with vasculature (Supplemental Figure 4). Therefore, in mesenchymal tumors, there is still only a small proportion of cancer cells that closely associate with blood vessels.

EMT cancer cells express pericyte markers in vitro and in vivo. The association of EMT cells with blood vessels apparently resembled that of pericytes. Pericytes are specialized mesenchymal cells that coat and stabilize the endothelium of small vessels. Pericytes are generally defined based on a combination of mesenchymal morphology, perivascular location, and expression of 2 or more pericyte markers (although there are no strictly pericyte-specific markers) (25-28). We asked whether EMT cancer cells might express pericyte markers. We previously performed microarray analysis to compare gene expression between neuT and neuTemt cells (24). To test whether EMT cells and pericytes were similar, we further compared these cells with mouse primary pericytes (29). Indeed, neuTemt cells displayed gene expression strikingly similar to that of pericytes (Supplemental Figure 5). Genes known to be expressed or function in pericytes, such as Pdgfrb, vimentin, N-cadherin, Vcam1 (vascular cell adhesion molecule-1, or Cd106), Itga5 (integrin $\alpha 5$ ), and AXL receptor tyrosine kinase (25-28), were highly expressed in neuTemt but not neuT cells (Supplemental Figure 5).

Quantitative RT-PCR and immunofluorescence staining confirmed that expression of many common pericyte markers was markedly elevated in neuTemt, Hs578T, and 786-O mesenchymal cells compared with neuT and MCF7 epithelial cells (Figure 3A, 
A

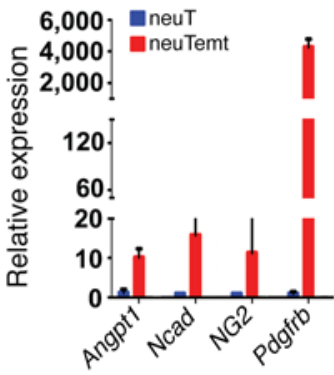

B

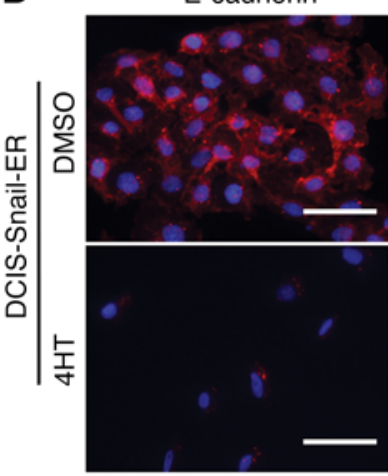

C

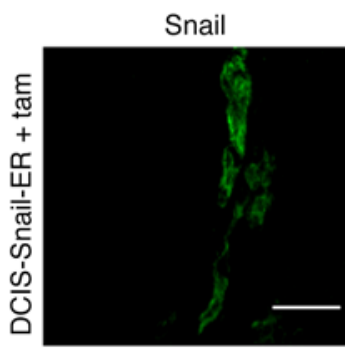

D

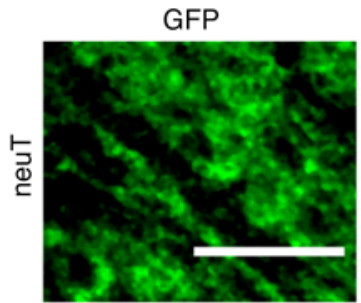

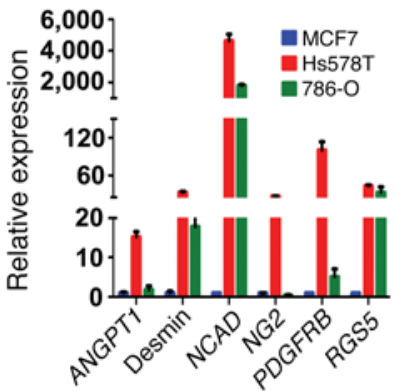

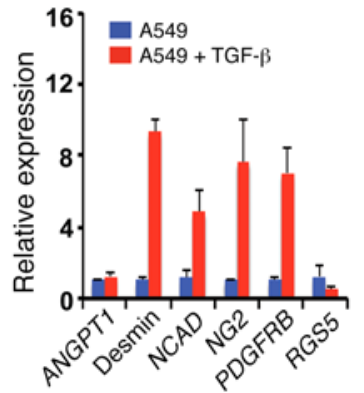

SMA
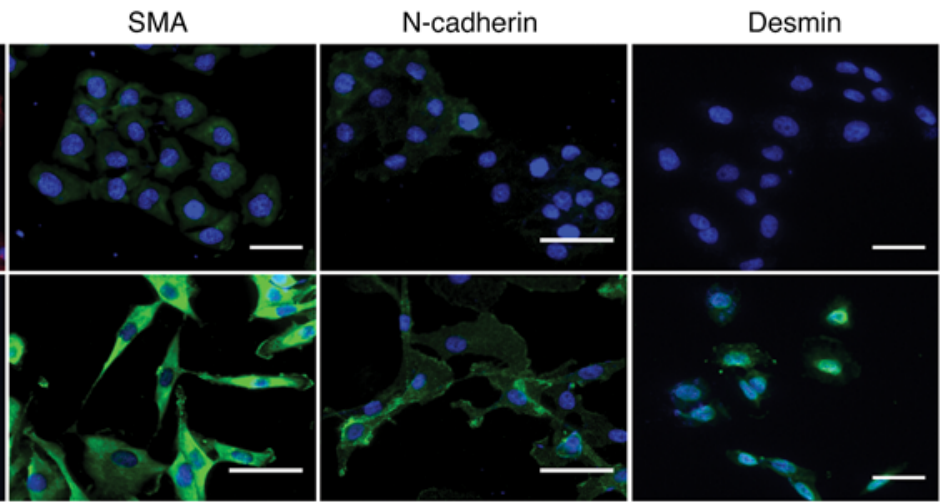

NG2
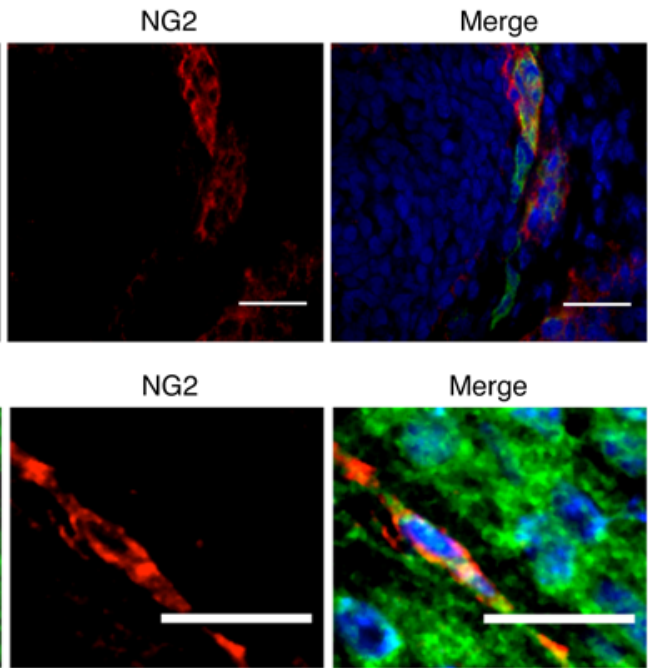

Figure 3. EMT cancer cells express pericyte markers in vitro and in vivo. (A) Quantitative RT-PCR analysis of expression of indicated pericyte markers in various epithelial, mesenchymal, and EMT cells. Error bars represent SD $(n=3)$. (B) Immunofluorescence staining of indicated EMT and pericyte markers in DCIS-SnailER cells that were treated with DMSO or 4 HT. $\alpha$-Smooth muscle actin (SMA) is a marker for a subpopulation of pericytes and smooth muscle cells. Scale bars: $50 \mu \mathrm{m}$. (C) Inducible EMT cancer cells express pericyte marker NG2 in vivo. DCIS-Snail-ER cells were mixed with DCIS cells ( $1: 4$, as in Figure 1) for tumor transplantation. Tumor-bearing mice were treated with tamoxifen (tam). Tumor sections were fixed with acetone, followed by immunostaining with anti-NG2 and anti-Snail antibodies for pericytes and DCIS-Snail-ER cancer cells, respectively. Scale bars: $50 \mu \mathrm{m}$. (D) Spontaneous EMT cancer cells express the pericyte marker NC2 in vivo. GFP-tagged neuT epithelial tumor cells were orthotopically implanted in mice. Tumor sections were fixed with acetone and immunostained with anti-NG2 antibody (for pericytes) and anti-GFP antibody (for tumor cells). Scale bars: $25 \mu \mathrm{m}$.
Supplemental Figure 6, A and B). TGF- $\beta$ is a potent EMT-inducing signal (20,30). Treatment of A549 human lung epithelial cancer cells with TGF- $\beta$ instigated typical EMT changes (31). Multiple pericyte markers were strongly upregulated by TGF- $\beta$ (Figure 3A). In the DCIS-Snail-ER inducible EMT model, expression of pericyte markers was increased while expression of E-cadherin was concurrently decreased by $4 \mathrm{HT}$ treatment (Figure $3 \mathrm{~B}$ ). These results suggest that EMT induces pericyte marker expression in epithelial cancer cells in vitro.

We asked whether EMT cancer cells express pericyte markers in vivo. In tumor xenografts derived from DCIS-Snail-ER and DCIS mixed cancer cells (as in Figure 1), following tamoxifen treatment, the majority of DCIS-Snail-ER cells expressed NG2 (Figure 3C and Supplemental Figure 7), which is one of the most commonly referenced pericyte markers (32). Consistent with this observation, while few ECs in tumor xenografts without tamoxi- fen treatment were associated with pericytes, more than $70 \%$ of ECs in tamoxifen-treated tumors became covered by NG2-positive cells (Supplemental Figure 8). These data suggest that inducible EMT cancer cells acquire pericyte marker expression in vivo.

We previously observed that a subset of neuT mammary epithelial tumor cells could undergo spontaneous EMT in vivo (24). We stably labeled neuT tumor cells with GFP through lentiviral transduction and generated tumor xenografts. Fluorescence microscopic examination of tumor sections revealed a small population of neuT cells displaying an elongated cell morphology, indicative of EMT (Supplemental Figure 9). Immunostaining of ECs demonstrated that such cells were adjacent to endothelial vasculature (Supplemental Figure 9), suggesting that spontaneous EMT cancer cells are also associated with blood vessels. These elongated tumor cells exhibited NG2 expression (Figure 3D). We further investigated whether a similar phenomenon might occur 
A Cancer cells

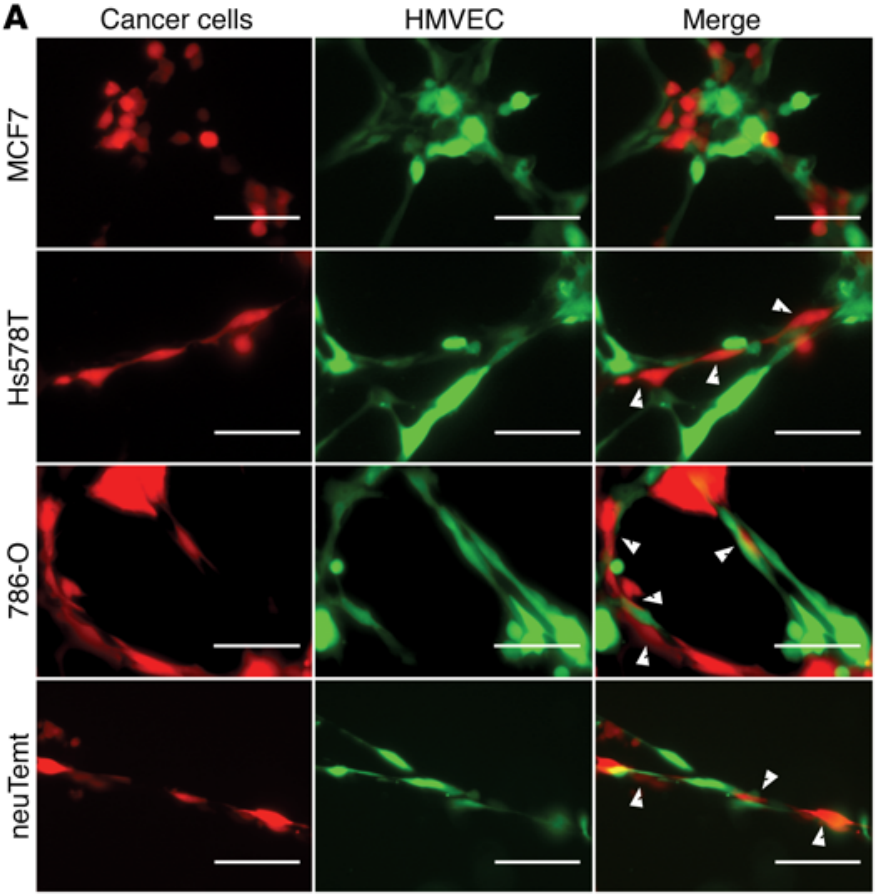

B

\section{B DCIS-Snail-ER}
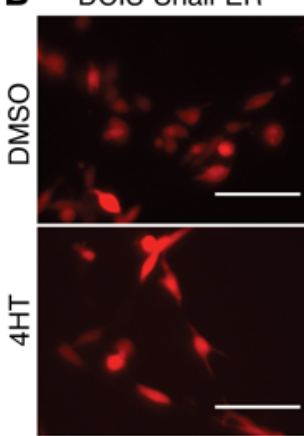

C
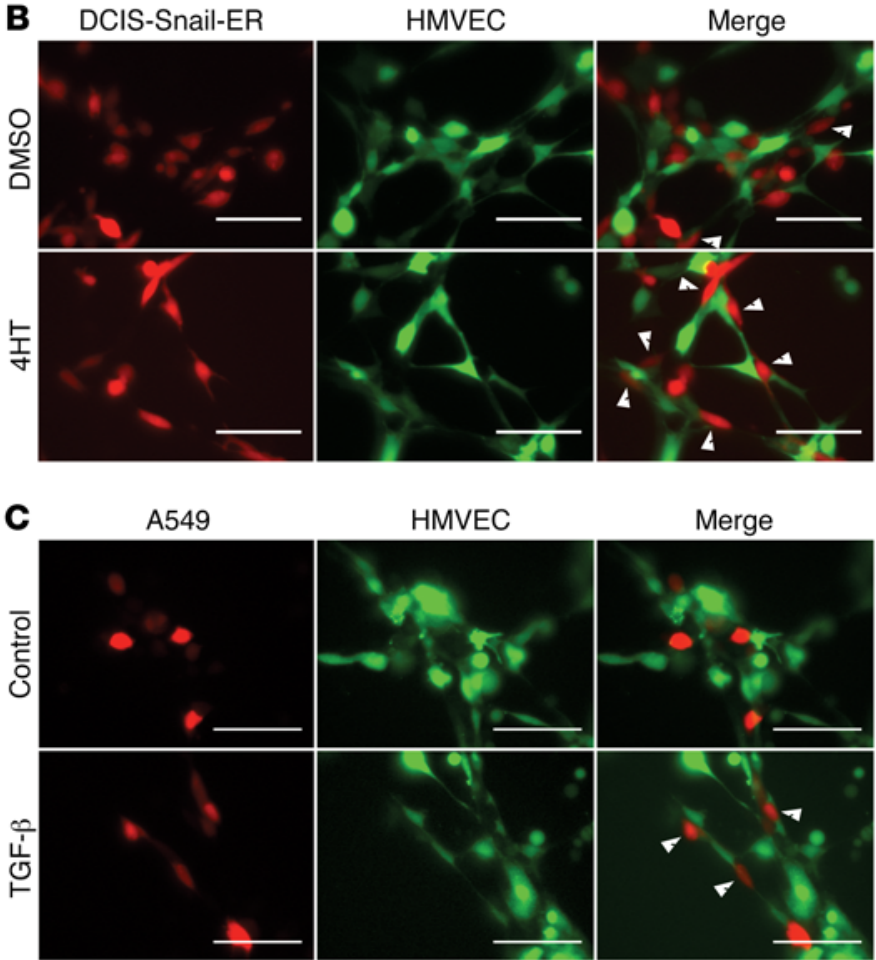

HMVEC
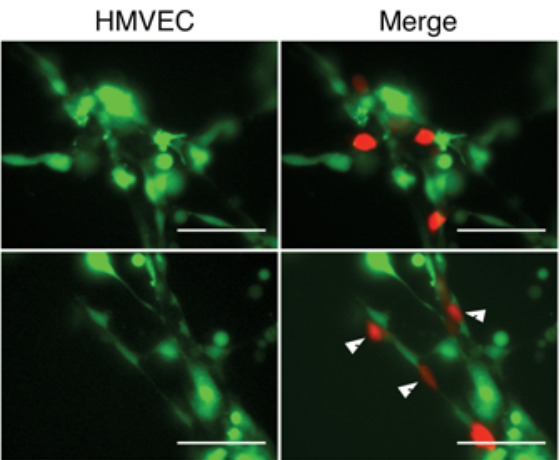

in human cancer. In a HER2-positive human breast carcinoma, simultaneous immunostaining of markers for cancer cells, pericytes, and ECs identified breast cancer cells that were attached to endothelium and expressed the pericyte marker $\alpha$-smooth muscle actin (SMA, encoded by Acta2 in mice) (Supplemental Figure 10). Taken together, these results suggest that spontaneous EMT cancer cells express pericyte markers in vivo.

EMT cells interact with ECs in vitro. Pericytes play an important role in regulation of EC proliferation. Pericyte coverage of the vas-

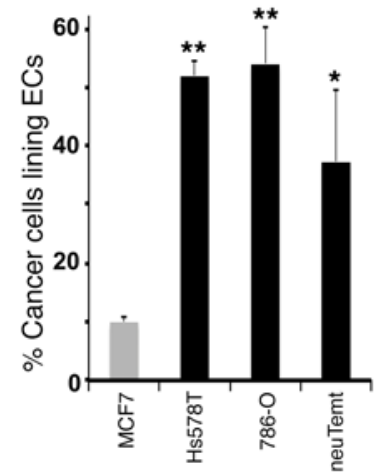

Figure 4. Mesenchymal/EMT cancer cells interact with endothelial cells in a coculture tube formation assay. Red-colored epithelial or mesenchymal cancer cells (A), DCIS-Snail-ER cells (pretreated without or with $4 \mathrm{HT}$ for 2 days) (B), and A549 cells (preincubated without or with TCF- $\beta$ for 2 days) (C) were mixed with green-colored HMVECs and coplated on Matrigel for tube formation. Photographs were taken 6 hours after seeding. Cancer cells exhibiting extensive interactions with $\mathrm{ECS}$ are indicated by white arrowheads and quantified (right panels). Scale bars: $50 \mu \mathrm{m}$. Error bars represent SD $(n=4-6) .{ }^{*} P<0.05,{ }^{*} P<0.01$. Statistical differences were determined by 2-tailed Student's $t$ test.
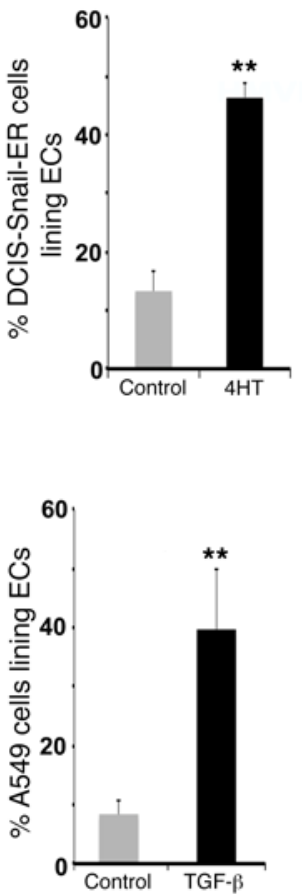

culature is associated with suppression of endothelial growth (25, $26,28)$. When endothelial cells were cocultured with epithelial or mesenchymal cancer cells, Hs578T and 786-O mesenchymal cells exhibited stronger inhibitory effects on endothelial proliferation than MCF7 epithelial cells (Supplemental Figure 11).

Pericytes can directly interact with ECs. These heterotypic cellcell interactions have been demonstrated in vitro in a 3D coculture tube formation assay $(33,34)$. Because EMT cancer cells expressed pericyte markers and associated with ECs in tumor xenografts, we 
A

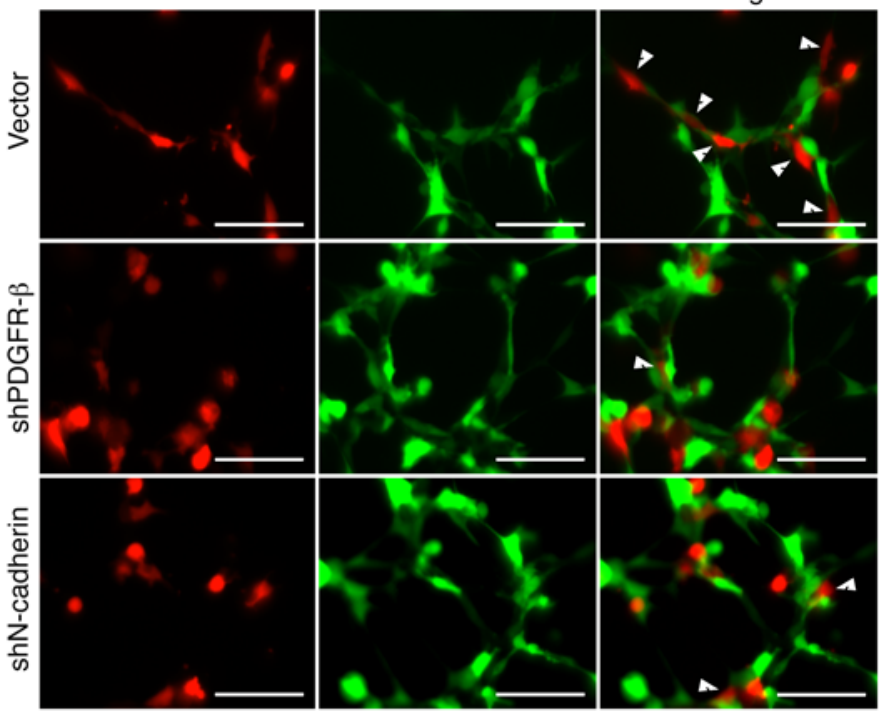

B
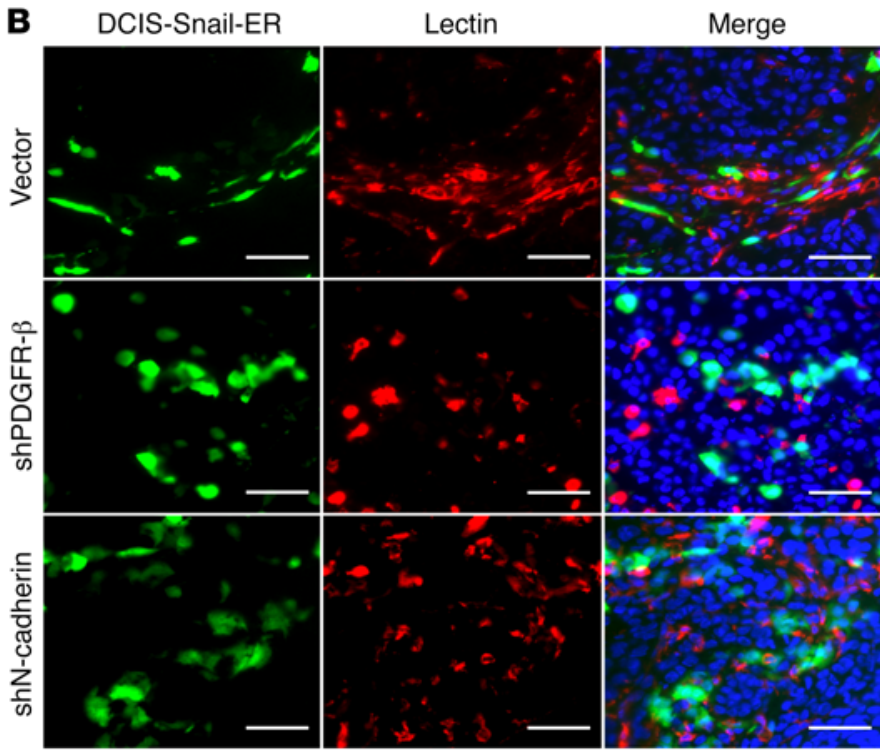

tested whether they could interact with ECs in vitro. Human microvascular endothelial cells (HMVECs) (35) and various cancer cells were differentially labeled with lentiviral GFP or red fluorescent protein (RFP) to distinguish the 2 cell types, mixed, and immediately coplated on Matrigel to form tube-like structures. More than $50 \%$ of mesenchymal Hs578T and 786-O cells, and nearly $40 \%$ of neuTemt cells stretched along HMVECs and exhibited tight adhesion to EC tubes in this coculture assay (Figure 4A). By contrast, MCF7 epithelial cells exhibited a round cell shape and less than $10 \%$ of them were able to tightly attach to ECs like mesenchymal cells (Figure 4A).

To test whether the affinity for ECs could be acquired through EMT, DCIS-Snail-ER cells with or without $4 \mathrm{HT}$ treatment were cocultured with ECs. Approximately $45 \%$ of 4 HT-treated cells displayed an elongated morphology and close association with EC capillaries, whereas less than $15 \%$ of control cells tightly adhered to ECs (Figure 4B). In the TGF- $\beta$-induced EMT model, around $40 \%$ of TGF- $\beta$-treated A549 cells demonstrated extensive interactions with ECs, compared with less than $10 \%$ of untreated A549
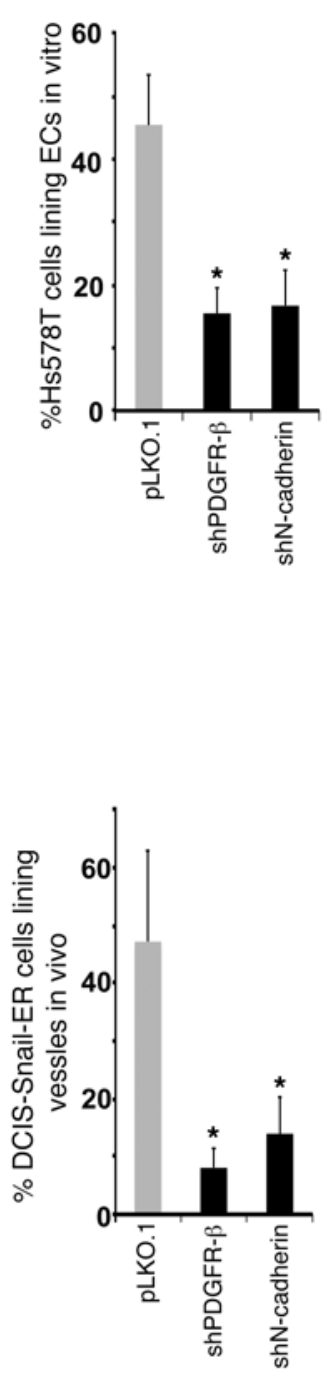

cells showing a similar pattern (Figure 4C). These findings suggest that EMT enables cancer cells to interact with ECs.

PDGFR- $\beta$ and $N$-cadherin expressed by EMT cancer cells are required for their association with vasculature in vitro and in vivo. The association of EMT cancer cells with ECs both in vivo and in vitro prompted us to identify molecules that were responsible for such heterotypic cell interactions. During normal vessel maturation, ECs secrete the PDGF ligand to chemoattract pericytes that express its cognate receptor, PDGFR- $\beta$, and this paracrine signaling plays a central role in pericyte recruitment and vascular stabilization (25, $26,28)$. Once recruited to the abluminal surface of endothelium, pericytes make direct peg-and-socket contact or form adhesion plaques with ECs (27). Expressed on plasma membranes of both cell types, $\mathrm{N}$-cadherin establishes adherens junctions that strengthen the heterotypic pericyte-EC association $(27,36,37)$.

We validated that PDGFR- $\beta$ expressed in mesenchymal cancer cells could be activated by PDGF-B or EC-conditioned media (Supplemental Figure 12A), suggesting that it is functional. As 
A
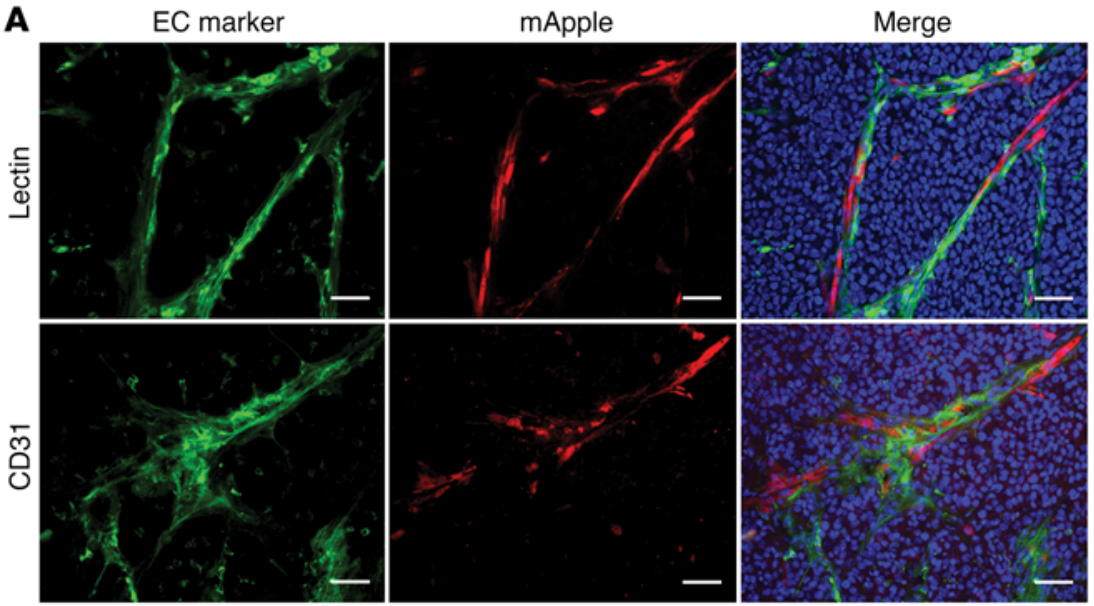

B
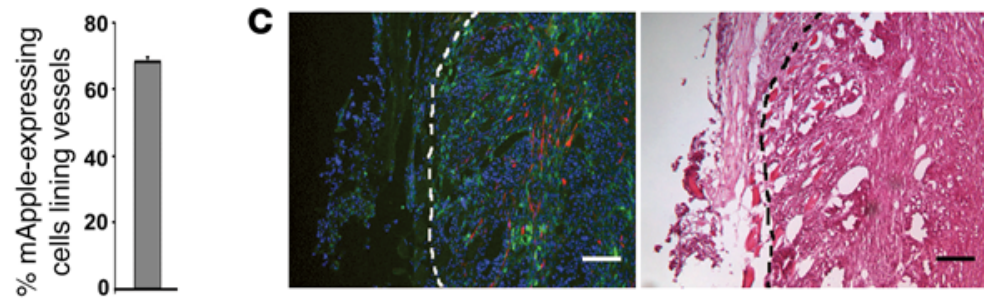

D
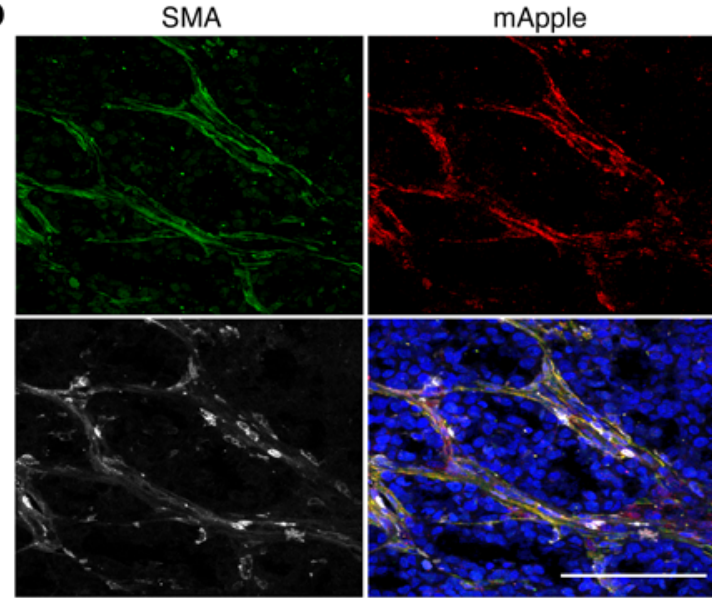

Lectin

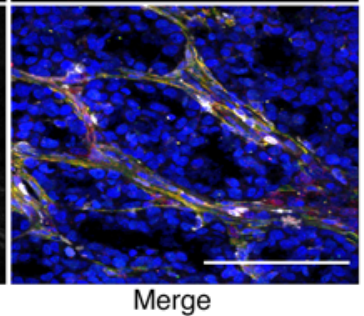

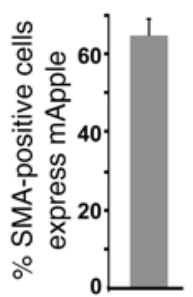

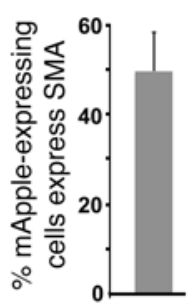

Figure 6. Spontaneous EMT cancer cells are associated with blood vessels in tumor xenografts and constitute a substantial portion of tumor pericytes. (A) NeuT tumor cells were transduced with the SMAmApple reporter and orthotopically transplanted into mice. Tumors were sectioned. A subpopulation of neuT cells underwent spontaneous EMT in vivo and thus expressed mApple (red). Endothelium was immunostained with anti-CD31 antibody (green). To label functional vasculature, tumor-bearing mice were injected with fluorescent Lycopericon esculentum lectin (green) via the tail vein prior to tumor harvest. Scale bars: $50 \mu \mathrm{m}$. (B) Quantification of mApple-expressing neuT tumor cells that are associated with blood vessels in tumor xenografts. Error bars represent SD $(n=4)$. (C) Spontaneous EMT cancer cells are not enriched at tumor edges. Green fluorescent lectin-perfused tumors from $\mathbf{A}$ were sectioned and examined for the localization of mApple-expressing cells (red). Scale bars: 100 $\mu \mathrm{m}$. (D) Spontaneous EMT cancer cells contribute to tumor pericytes. Lectin-perfused SMA-mApple neuT tumors from $A$ were sectioned and immunostained for pericytes with anti-SMA antibody (pseudocolored, green). Lectin-marked vasculature was pseudocolored white. Spontaneous EMT cells expressed mApple (red) Scale bar: $100 \mu \mathrm{m}$. Histograms (right) show the substantial overlap between SMA-positive pericytes and mApple-expressing EMT tumor cells $(n=4)$.
PDGFR- $\beta$ and N-cadherin were upregulated in EMT cells (Figure 3 and Supplemental Figure 5), we tested whether these 2 molecules were required for the observed interactions between EMT cancer cells and ECs. We depleted PDGFR- $\beta$ and N-cadherin in Hs578T mesenchymal cancer cells using lentiviral short hairpin RNAs (shRNAs) (Supplemental Figure 12B). PDGFR- $\beta$-depleted Hs578T cells slightly upregulated epithelial genes and showed reduced chemotaxis towards ECs compared with control Hs578T cells (Supplemental Figure 12, C and D). In the EC coculture tube formation assay, while approximately $45 \%$ of control Hs $578 \mathrm{~T}$ cells exhibited intimate association with ECs, only $15 \%$ of PDGFR- $\beta$ - or $\mathrm{N}$-cadherin-depleted cells remained closely bound to ECs (Figure $5 \mathrm{~A})$, suggesting that both PDGFR- $\beta$ and $\mathrm{N}$-cadherin expressed by cancer cells are necessary for the EMT cell-EC interaction in vitro.

To verify the role of PDGFR- $\beta$ and N-cadherin in EMT cell$\mathrm{EC}$ association in vivo, we depleted them in GFP-tagged DCIS-
Snail-ER cells (Supplemental Figure 12B), and mixed the cells with unlabeled DCIS for orthotopic implantation. All tumor-bearing mice were treated with tamoxifen to induce EMT in DCIS-SnailER cells. In tumor sections, nearly $50 \%$ of control DCIS-Snail-ER cells displayed tight association with vascular ECs; however, most PDGFR- $\beta$-depleted DCIS-Snail-ER cells were detached from vasculature, and less than $10 \%$ of them exhibited close interactions with ECs (Figure 5B). While N-cadherin-depleted DCIS-SnailER cells were found in the vicinity of blood vessels, they lost the ability to align with ECs (Figure 5B). These observations suggest that PDGFR- $\beta$ and $N$-cadherin expressed by EMT tumor cells are required for their association with ECs in vivo.

Spontaneous EMT cancer cells are associated with vasculature and significantly contribute to tumor pericytes. Based on their pericyte marker expression, perivascular localization, and EC interactions, EMT and mesenchymal cancer cells strongly resembled pericytes. 
A
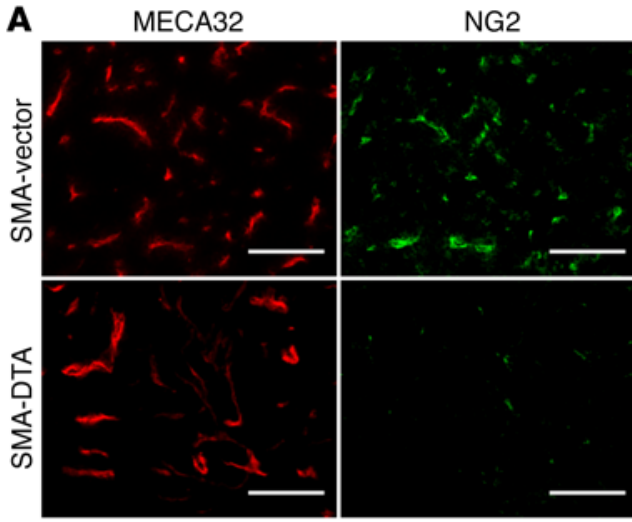

CD34
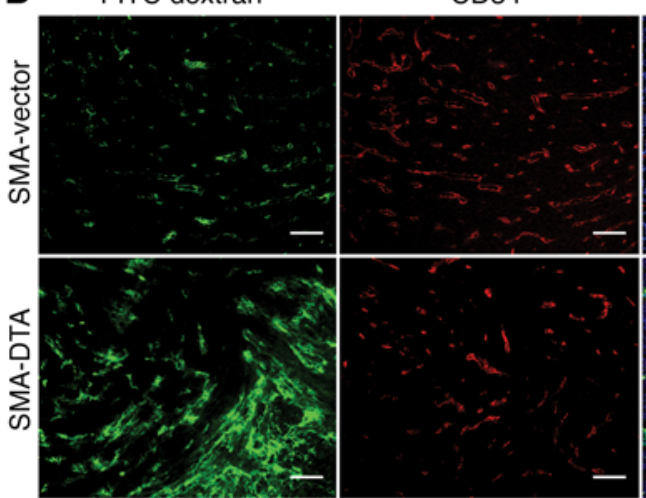

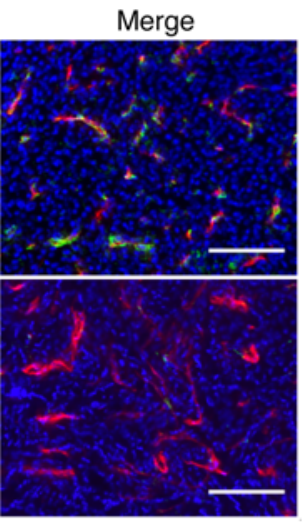

Merge
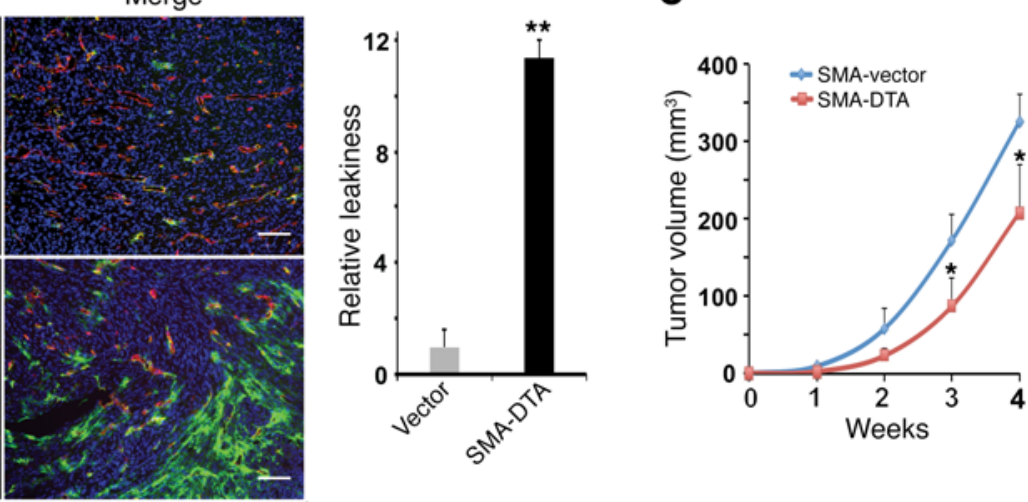

D

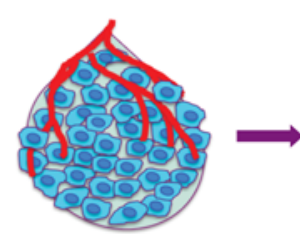

Tumor with nascent, leaky endothelial blood vessels

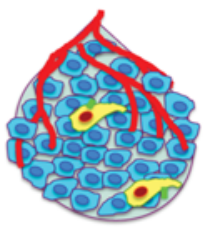

A subset of tumor cells undergo EMT, express PDGFR- $\beta$ and $\mathrm{N}$-cadherin

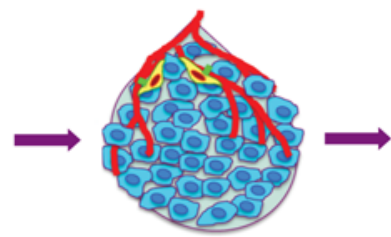

EMT cancer cells are chemotactically attracted to and interact with endothelium

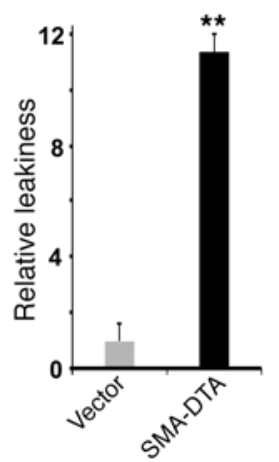

C
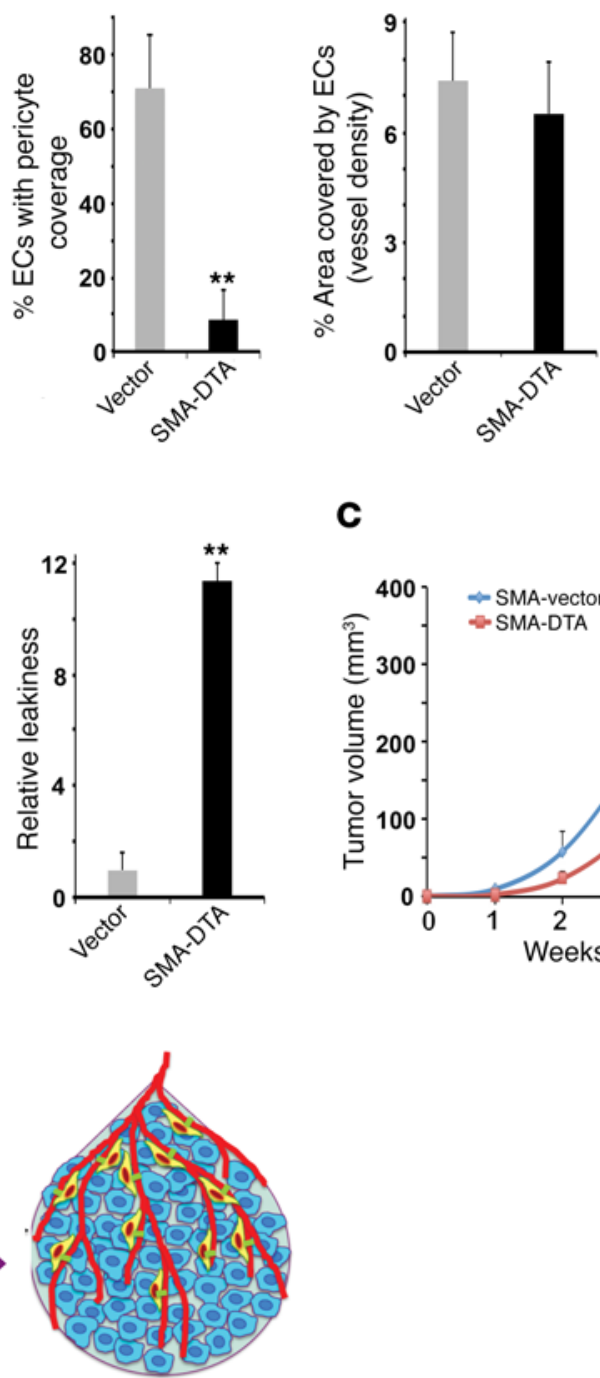

Like pericytes, EMT cancer cells stabilize blood vessels to fuel bulk tumor growth

Figure 7. Spontaneous EMT cancer cells are essential for pericyte coverage and integrity of tumor vasculature. NeuT tumor cells were infected with lentiviral control vector or SMA-DTA to eliminate EMT cells, and orthotopically injected into mice. Statistical differences were determined by 2-tailed Student's $t$ test. (A) Pericyte coverage in tumor sections was determined by immunostaining of pericytes with anti-NC2 antibody and ECs with anti-MECA32 antibody. Scale bars: $50 \mu \mathrm{m}$. Histograms (right) of pericyte coverage and vessel density in control and SMA-DTA neuT tumors. Error bars represent SD $(n=5)$. ${ }^{* *} P<0.01$. (B) Tumor vascular permeability was visualized by intravenously infusing tumor-bearing mice with FITC-dextran dye (green). Tumor sections were stained for ECs with anti-CD34 antibody. Scale bars: $100 \mu \mathrm{m}$. Quantification of relative vascular leakiness is on the right. Error bars represent SD $(n=4) .{ }^{*} P<0.01$. (C) Growth rate of control and SMA-DTA neuT tumors. Tumor volumes were measured at indicated time points. Error bars represent SD $(n=5) .{ }^{*} P<0.05$. (D) A schematic model for the behavior and function of EMT carcinoma cells in tumor growth.

A key question is whether carcinoma cells that had undergone spontaneous EMT could contribute to functional pericytes in tumors. The rates of spontaneous EMT in cancer cells in vivo are largely unknown. To better detect spontaneous EMT cells, we sought to establish a reporter system that could highlight such cells. As the Acta2 promoter was active only in mesenchymal cells in a previous study (38), we used it to drive the expression of the mApple fluorescent reporter in a lentiviral vector. When transduced into cells, the Acta2-mApple reporter (hereafter referred to as the SMA-mApple reporter) was active in neuTemt mesenchymal cells but not neuT epithelial cells (Supplemental Figure 13A). When neuT cells were treated with TGF- $\beta$ to undergo EMT, the reporter became activated (Supplemental Figure 13A). Similarly, the reporter was inactive in A549 epithelial lung cancer cells, and was activated following TGF- $\beta$-induced EMT (Supplemental Figure 13B).

Prolonged culture of neuT epithelial cells in media containing penicillin and streptomycin led to spontaneous EMT in vitro. Accordingly, in neuT cells transduced with the SMA-mApple 
reporter, a subset of infected cells started expressing mApple following extended culture in vitro. Immunofluorescence analysis confirmed that these mApple-expressing neuT cells had lost E-cadherin and acquired expression of N-cadherin and SMA (Supplemental Figure 14), suggesting that the SMA-mApple reporter can reliably mark spontaneous EMT cells.

Spontaneous EMT occurs in neuT tumor xenografts (Supplemental Figure 9) (24). To track tumor cells that spontaneously underwent EMT in vivo, we orthotopically transplanted SMAmApple-transduced neuT epithelial cells into mice (prior to transplantation, potential preexisting mApple-expressing EMT cells were removed by flow cytometry). In the resulting tumor xenografts, a fraction ( $14 \%$ on average) of neuT tumor cells activated mApple expression, suggesting that neuT epithelial cells indeed undergo frequent spontaneous EMT in vivo. Similar to aforementioned EMT cells and mesenchymal cancer cells, the mAppleexpressing neuT cells formed vascular-like structures (Figure 6A and Supplemental Figure 15). Immunostaining of EC markers CD31 and VEGFR2 demonstrated that the mApple-expressing neuT cells tightly adhered to endothelial vasculature (Figure 6A and Supplemental Figure 15). To validate that these blood vessels were functional, tumor-bearing mice were injected with fluorescent lectin dye via the tail vein prior to harvesting tumors. Consistently, mApple-expressing neuT cells were found to be juxtaposed with lectin-labeled functional vasculature (Figure 6A and Supplemental Figure 15). Overall, approximately $70 \%$ of the mAppleexpressing neuT cells were intimately associated with blood vessels (Figure 6B). Although EMT was presumed to occur at the invasive front of primary tumors, the mApple-expressing neuT cells were not enriched at the edge of tumors (Figure 6C).

To determine whether spontaneous EMT cancer cells contributed to tumor pericytes, we stained pericytes in tumors with anti-SMA antibodies. Over $60 \%$ of SMA-positive cells were indeed derived from mApple-expressing neuT cells (Figure 6D and Supplemental Figure 16). Such cells were also associated with functional blood vessels (marked by intravenously injected lectin) (Figure 6D), indicating that they are putative pericytes. Collectively, these results suggest that spontaneous EMT cancer cells are mostly associated with blood vessels and may constitute a substantial portion of all pericytes in tumors.

Spontaneous EMT cancer cells are essential for stabilization of tumor blood vessels and tumor growth. Pericytes are obligatory for vascular integrity. As EMT cancer cells simulated pericytes, we asked whether such cancer-derived cells perform pericyte function and are important for tumor vasculature. For this purpose, we depleted spontaneous EMT cells in tumors and assessed the consequence for tumor blood vessels. We generated a lentiviral construct using the Acta2 promoter to drive a suicide gene, diphtheria toxin fragment A (DTA), which has been widely used to ablate cells in vivo $(39,40)$. Mesenchymal cells transduced with the lentiviral Acta2-DTA construct (hereafter referred to as SMA-DTA) died from the cytotoxic DTA. We infected neuT epithelial cells with the lentiviral SMA-DTA or a control vector, and implanted the cells in mice. To evaluate the pericyte coverage in tumors, we stained pericytes with anti-NG2 antibodies and ECs with MECA32 antibodies (41). Consistent with the early observation that neuT tumor vasculature was well coated by mural cells
(Supplemental Figure 1B), around 70\% of ECs in control neuT tumors were covered by NG2-expressing cells (Figure 7A). By contrast, the vast majority of blood vessels in the SMA-DTA neuT tumors lacked pericytes, and the pericyte coverage was below $10 \%$ (Figure 7A). The decrease in tumor pericytes was also confirmed by quantification of pericyte marker expression (Supplemental Figure 17). The vessel density (based on EC staining) was not significantly different between the tumors (Figure 7A). These results suggest that elimination of EMT cancer cells diminishes pericyte coverage in tumors, which is consistent with the result showing that most SMA-expressing cells in neuT tumors originate from spontaneous EMT tumor cells (Figure 6D).

Deficient pericyte coverage destabilizes vasculature (25, 26). Therefore, we surveyed vascular permeability in tumors through intravenous injection of the FITC-dextran dye into tumor-bearing mice. Leaky blood vessels would allow the fluorescent dextran tracers to leak out of the capillaries. Consistent with the pericyte coverage, control neuT tumors exhibited intact vasculature with negligible leakiness, which was evidenced by generally vessel-confined distribution of the dextran dye (Figure 7B). By contrast, in SMA-DTA neuT tumors, there was a massive increase in extravascularly diffused dextran (Figure 7B), indicative of hyperpermeable blood vessels. Tumor growth critically relies on effective vascular support for access to nutrients and oxygen and removal of metabolic waste $(25,26)$. Leaky blood vessels are dysfunctional and ineffective in sustaining tumor growth. SMA-DTA neuT tumors indeed grew more slowly than control neuT tumors (Figure 7C). Taken together, these results suggest that through spontaneous EMT, cancer cells functionally resemble pericytes and are required to stabilize tumor blood vessels and fuel tumor growth (Figure 7D).

\section{Discussion}

The attributes of EMT cancer cells have led to the popular hypothesis that EMT critically drives tumor metastasis. However, this notion has been a matter of debate. Recent studies show that EMT does not contribute to spontaneous tumor metastasis in vivo (16-18). Therefore, the significance of EMT in cancer as well as the fate and function of mesenchymal-switched cancer cells in vivo remain poorly defined. We tracked epithelial and mesenchymal cancer cells derived from various tissues as well as inducible and spontaneous EMT breast cancer cells in tumor transplantation models. We found that EMT cancer cells are not located at the tumor-stroma interface. Instead, the majority of them exhibit intratumoral perivascular localization and close association with blood vessels, thus resembling pericytes. EMT markedly activates pericyte markers in epithelial cancer cells, and enables them to interact with ECs in vitro and in vivo. For carcinoma cells that tend to undergo EMT, EMT cancer cells may become a significant contributor to pericytes associated with tumor vasculature, and are indispensable for pericyte coverage and vascular integrity in tumor xenografts. These findings uncover an unorthodox role for EMT in cancer: EMT confers key pericyte properties on carcinoma cells. EMT cancer cells in tumor mass may assume the identity of pericytes (i.e., disguised as stromal cells) and perform pericyte function to stabilize tumor vasculature, thereby improving vascular support for the growth of the bulk tumor (Figure 7D). 
Tumor vasculature is often portrayed as poorly organized, constantly remodeling, and lacking appreciable pericyte coverage (42). However, microscopic studies have revealed the nearly ubiquitous presence of pericytes on tumor vessels, although such pericytes are more loosely attached to the vasculature and at reduced density compared with respective normal tissues (25, 43-45). Different cancer cells exhibit dramatically varying pericyte coverage when transplanted to form tumor xenografts (46). In our study, DCIS cell-derived tumor xenografts had very few pericytes whereas blood vessels in neuT tumors were well covered by pericytes (Figure 7A and Supplemental Figures 1B and 8). Importantly, tumor vasculature-associated pericytes are functional, as pharmacological blockade of pericyte recruitment through inhibition of PDGFR- $\beta$ reduces pericyte coverage, destabilizes blood vessels in vivo, and decreases tumor growth $(25,42,47)$. Our study suggests that like pericytes, EMT cancer cells critically stabilize nascent vasculature to nourish tumor growth. In addition, pericytes provide important paracrine trophic signals (including VEGF) to support EC survival and protect ECs from antiangiogenic treatment $(25,48)$. Pericyte coverage accounts for the relative resistance of more mature vessels to VEGF withdrawal. Therefore, EMT may also confer resistance to VEGF-targeted antiangiogenic therapies.

PDGFR- $\beta$ and $N$-cadherin are critically implicated in the pericyte-EC interactions (25-28). In carcinomas, expression of PDGFR- $\beta$ is generally restricted to stromal cells of mesenchymal origin, and is absent in epithelial tumor cells (49). However, EMT almost universally upregulates PDGFR- $\beta$ expression in carcinoma cells $(24,50-54)$ (and this study), which may allow EMT cancer cells to respond to EC-secreted chemoattractant PDGF-B and be recruited towards blood vessels. This may explain the observed location of EMT cancer cells at intratumoral vasculature instead of tumor edge. The PDGF-B signaling may also keep EMT tumor cells in the mesenchymal state (53). N- and E-cadherins usually exhibit mutually exclusive expression patterns, with E-cadherin primarily expressed in epithelial cells and N-cadherin in mesenchymal cells and ECs (55). As classical cadherins exhibit preferentially homophilic interactions, epithelial cells are unable to form adherens junctions with ECs. The E-cadherin-to-N-cadherin switch is a hallmark of EMT $(3,56)$. As homotypic N-cadherin interactions are weaker than homotypic E-cadherin interactions, and $\mathrm{N}$-cadherin signaling stimulates cell motility, EMT-mediated cadherin switch facilitates cell migration and invasion (55). Furthermore, through this switch, the transitioning cells acquire an affinity for ECs through homotypic N-cadherin interactions. Collectively, the data show that EMT allows carcinoma cells to detach from adjoining neighbors, migrate to the proximity of vasculature through PDGF-mediated chemotaxis, and attach to ECs via $\mathrm{N}$-cadherin-mediated adhesion. Because the induction of PDGFR- $\beta$ and N-cadherin is a prevalent feature of EMT, EMT may generally confer key pericyte properties on epithelial cells, and may hence represent epithelial-to-pericyte transition under certain circumstances. Indeed, during mammalian embryonic development, many normal pericytes result from EMT (26). Pericytes of the head, thymus, and outflow tract of the aorta are mostly derivatives of the neural crest, which represents a classical model of EMT, and pericytes in the internal viscera originate from mesothelial cells that undergo EMT (26). Previously, certain malignant melanoma and glioma cells were found to associate with blood vessels "without any evidence of intravasation" (57). Melanoma is derived from melanocyte transformation and tends to reactivate the EMT program that has enabled their neural crest ancestors to migrate during embryonic development. Malignant gliomas are often mesenchymal (58). It is plausible that these vascular-associating melanoma and glioma cells are post-EMT cells. More recently, glioma stem cells were shown to be able to differentiate into pericytes (59). The differentiation process is induced by TGF- $\beta$, which may also activate the EMT program as well. Overall, EMT may significantly contribute to the development of both normal and tumor pericytes.

Other than stimulating angiogenesis, aggressive cancer cells can form de novo perfusable, vascular-like networks by themselves, a phenomenon known as vascular mimicry (60). Recent studies show that subsets of glioma and melanoma cells (especially stem-like tumor cells) are able to switch on certain EC markers and transdifferentiate into vascular ECs (61-65). Such tumor-derived ECs participate in vascularization to ensure a steady supply of oxygen and nutrients for tumor growth. Although we found that EMT cancer cells resemble pericytes and do not evidently contribute to ECs, these studies collectively reflect the complexity, heterogeneity, and importance of the tumor vasculature.

\section{Methods}

Cell culture. Human MCF7, Hs578T, A549, H358, 786-O, and HCT116 cancer cells were obtained from ATCC. Mouse neuT and neuTemt mammary tumor cells were previously established (24). All cells (except 786-O and neuT) were cultured in Dulbecco's Modified Eagle's Medium (DMEM) supplemented with $10 \%$ fetal bovine serum (FBS) and penicillin-streptomycin. MCF10DCIS cells (Asterand) were maintained in DMEM/F12 supplemented with $10 \%$ horse serum, and 786-O cells were in RPMI with $10 \%$ FBS. NeuT cells were maintained in media without antibiotics. HMVECs were cultured in EBM2 media with growth factor supplement.

RNA extraction and real-time RT-PCR. Total RNA was extracted using TRIzol (Invitrogen) from various cell lines and indicated tumor samples in accordance with the manufacturer's protocol. cDNA was generated using $1.5 \mu$ g of total RNA. Real-time PCR was carried out using SYBR green detection reagents (Applied Biosystems). Quantitative RT-PCR was conducted in triplicate; data represent the mean $\pm \mathrm{SD}$. The primers used in the study were: hANGPT1, (sense) 5'-GTCAATGGGGGAGGTTGGACTGT-3' and (antisense) 5'-TAGGCTCGGTTCCCTTCCCAGT-3'; hDesmin, (sense) 5'-GAGGAGGAGCTGCGGGAGCT-3' and (antisense) 5'-CAGCTTGGCCTTGAGCCGCT-3'; hN-cadherin, (sense) 5'-TCAGGCTCCAAGCACCCCTTCA-3' and (antisense) 5'-ATGACGGCCGTGGCTGTGTT-3'; hNG2, (sense) 5'-CTAGGCCGGCTGTTCCACGC-3' and (antisense) 5'-GCCTCCCAAAAGGGCTCGGG-3'; hPDGFRB, (sense) 5'-CTGTGGGCCAGGACACGCAG-3' and (antisense) 5'-AGCACCACCAGGGCCAGGAT-3'; hRGS5, (sense) 5'-GGCCCTGCAGTGGCGTGATT-3' and (antisense) 5'-CAGCCATCTTGGCAGGGGACT-3'; mAngpt1, (sense) 5'-CAGCATCTGGAGCATGTGAT-3' and (antisense) 5'-TTCTGTTGTATCTGGGCCATC-3'; mN-cadherin, (sense) 5'-ACAGCGCAGTCTTACCGAAG-3' and (antisense) 5'-TGGCTCGCTGCTTTCATAC-3'; mNG2, (sense) 5'-GACACCCGCTGTCAGCTC-3' and (anti- 
sense) 5'-GCACCTCCAGGTGGTTCTC-3'; mPdgfrb, (sense) 5'-CGGAGCTCAGTGAGAGGAAG-3' and (antisense) 5'-TCCGTGACAAAAATGAACAGG-3'. As control, $\beta$-actin primers were used: (sense) 5'-ACTGGAACGGTGAAGGTGAC-3' and (antisense) 5'-AGAGAAGTGGGGTGGCTTTT-3' for human and (sense) 5'-CTGAACCCTAAGGCCAACCG-3' and (antisense) 5'-GAGTCCATCACAATGCCTGTG-3' for mouse. In the above-mentioned primers, $\mathrm{h}$ stands for human and $\mathrm{m}$ for mouse.

Coculture tube formation assay. Tube formation assay was performed using indicated cancer cell lines and HMVECs. HMVECs (infected with RFP lentivirus) and cancer cells (infected with GFP lentivirus) were used at a ratio of 3:1. DCIS-Snail-ER-GFP cells were treated with $4 \mathrm{HT}(100 \mathrm{nM})$ for 2 days and then used in coculture assay. A549 cells pretreated with TGF- $\beta$ ( $5 \mathrm{ng} / \mathrm{ml}$ ) for 2 days were then cocultured with HMVECs. All experimental conditions were set up and analyzed in triplicate. A total of 15,000 cells were suspended in EBM2 media (Lonza) and inoculated in 96-well plates containing 50 $\mu \mathrm{l}$ of growth factor-reduced Matrigel (BD Biosciences). Photographs of 4 random fields were taken at $\times 100$ magnification 4 hours after seeding. Fluorescent images were obtained using a Leica DMI 4000B microscope accompanied by Openlab software (PerkinElmer). HMVECs and cancer cells that aligned perfectly with one another were quantified manually using ImageJ software (NIH). Cancer cells that remained round were considered negative for EC interaction.

shRNA knockdown. Lentiviral vector pLKO.1 and shRNAs for $\mathrm{N}$-cadherin and PDGFR- $\beta$ (The RNAi Consortium) were transfected into 293FT packaging cells together with helper plasmids. Supernatant from transfected cells was filtered and used to infect indicated cell lines in the presence of $8 \mu \mathrm{g} / \mathrm{ml}$ polybrene. Infected cells were subsequently selected with $1 \mu \mathrm{g} / \mathrm{ml}$ puromycin.

Western blotting. Whole-cell lysates were resolved by gel electrophoresis using an $8 \%$ SDS-PAGE gel, electrotransferred to a PVDF membrane, and probed with anti-E-cadherin (Cell Signaling Technology, catalog 3195), anti-PDGFR- $\beta$ (Cell Signaling Technology, catalog 4564 ), anti-PDGFR- $\beta$ phospho-Y751 (Cell Signaling Technology, cata$\log 4549$ ), anti-N-cadherin (BD-Transduction Laboratories, catalog 610920), and anti-tubulin (Sigma-Aldrich, catalog T9026) antibodies.

Mouse tumor transplantation models. Inbred nonobese diabetic/ severe combined immunodeficient (NOD/SCID) mice (5-6 weeks old) were maintained under pathogen-free conditions. Tumors were generated by injecting $2 \times 10^{6}$ of various cancer cells in the flanks or mammary fat pad of NOD-SCID mice. Mice were euthanized once the tumors reached $1.5 \mathrm{~cm}$ in diameter. To induce EMT in DCIS-Snail-ER cells, tumor-bearing mice were randomly divided into 2 groups, and received intraperitoneal injection of tamoxifen $(2 \mathrm{mg} / 200 \mu \mathrm{l}$ dissolved in sesame oil) or sesame oil every 2 days. For depletion of spontaneous EMT tumor cells in vivo, neuT tumor cells were infected with SMAmApple (control) or SMA-DTA lentivirus. Mice received subcutaneous injection of $2 \times 10^{6}$ control cells or SMA-DTA cells. Tumor growth was monitored weekly using digital calipers.

Immunofluorescence analysis and quantification. For immunofluorescence of cultured cancer cells, cells were fixed with $4 \%$ paraformaldehyde for 20 minutes, permeabilized with $0.5 \%$ Triton X-100, and stained with anti-E-cadherin antibody (Cell Signaling Technology, catalog 3195), anti-N-cadherin antibody (Cell Signaling Technology, cata$\log$ 13116), anti-SMA antibody (Sigma-Aldrich, catalog SAB5500002), and anti-desmin antibody (R\&D Systems, catalog AF3844).
For immunostaining of tumor sections (except for anti-NG2 antibody), tumor xenografts were fixed in $4 \%$ paraformaldehyde and OCT embedded. Tumor sections (5- $\mu \mathrm{m}$ thick) were stained with Griffonia simplicifolia isolectin B4 (Sigma-Aldrich, catalog L2140), anti-CD31 (BD Pharmingen, catalog 550274), anti-CD34 (Biolegend, catalog 119301), anti-VE-cadherin (Cell Signaling Technology, catalog 2500), anti-VEGFR2 (Cell Signaling Technology, catalog 2479), anti-SMA (Sigma-Aldrich, catalog SAB5500002), and Cy3-conjugated antiSMA (Sigma-Aldrich, catalog C6198) antibodies.

For pericyte staining with anti-NG2 antibody, sections $(5-\mu \mathrm{m}$ thick) of fresh-frozen tumor samples were fixed with acetone. AntiNG2 (Millipore, catalog AB5320) and anti-MECA32 (Biolegend, cata$\log 120501)$ antibodies were used to stain pericytes and endothelial cells, respectively. DCIS-Snail-ER cells were stained with anti-Snail antibody (R\&D Systems, catalog AF3639).

Visualization was carried out under the same settings for comparison using a Leica DMI 6000 B microscope with Openlab software. Quantification in G. simplicifolia isolectin B4-stained tumors was carried out by counting the percentage of GFP-positive cells aligned with blood vessels in 4-6 random visual fields per tumor (3-5 tumors) at original magnifications of $\times 100$ or $\times 200$.

To visualize functional blood vessels in SMA-mApple neuT tumor xenografts, tumor-bearing mice were injected with Dylight 488 Lycopericon esculentum (tomato) lectin $(200 \mu \mathrm{g} / 200 \mu \mathrm{l})$ (Vector Laboratories, catalog DL-1174) via the tail vein 3 hours prior to tumor harvest. To determine the cell origin of pericytes, tumor sections were stained with a rabbit anti-SMA antibody (Sigma-Aldrich, cata$\log$ SAB5500002, 1:400 dilution) and Alexa Fluor 647-conjugated anti-rabbit secondary antibody. Confocal images were taken using a Leica TCS SP5 accompanied by LAS AF software. Spontaneous EMT cells express mApple (red). Dylight 488 lectin signal was pseudocolored white and Alexa Fluor 647 SMA pseudocolored green (Figure 6D). The percentage of SMA-positive cells overlapping with mAppleexpressing cells was counted manually using ImageJ software in $\times 200$ magnification fields.

Vascular leakiness and density measurement. Mice carrying neuT tumors at similar sizes were injected via the tail vein with $1 \mathrm{mg} / 200$ $\mu$ FITC-dextran (2,000 kDa, Sigma-Aldrich) 30 minutes before sacrifice. Tumor sections from OCT-embedded tissue were stained for ECs with anti-CD34 antibodies and visualized by fluorescence microscopy. As previously described (47), quantification of extravasation of FITC-dextran was performed using a grading system in which 0 represents 0\% extravascular FITC-dextran and 5 represents 100\% extravascular FITC-dextran. Results were plotted as fold change in extravascular FITC-dextran compared with controls. For blood vessel density, area of the blood vessel (labeled by anti-CD34 antibody) was calculated with Image (using the threshold and analyze tool).

Microarray gene expression analysis. Microarray datasets from neuT and neuTemt (cl2) cells (24) and mouse primary pericytes (NCBI GEO GSE46564) (29) were analyzed by clustering. Briefly, we identified differentially expressed genes (fold change $>2, P<0.05$ ) between neuT and neuTemt microarray datasets. Subsequently, we performed a gene expression cluster analysis among neuT, neuTemt, and pericyte microarray data based on these genes. We derived a multigene score as follows: normalized probeset $\log 2$ values were calculated with median values from all samples, and these data were uploaded into Cluster 3.0. We hierarchically clustered the genes using centered cor- 
relation and complete linkage as the distance metric and clustering method, respectively. The output data were loaded into Java Tree View software to generate heatmaps (66).

Statistics. Statistical differences between different experimental groups were determined by Student's $t$ test ( 2 tailed). The reported values represent the mean $\pm \mathrm{SD}$ as indicated in the figures. A $P$ value less than 0.05 was considered statistically significant. No samples were excluded from the analysis.

Study approval. All animal experimental procedures were approved by the University of Florida IACUC.

\section{Author contributions}

AKS designed and performed tumor transplantation experiments and most in vitro assays. YJ, HL, and MT participated in characterization of EMT cells in vitro. $\mathrm{CP}$ assisted with the tail vein injection experiments. RS, DWS, LW, CDH, BKL, and LJC provided reagents and/or suggestions. JL designed the study and wrote the manuscript.

\section{Acknowledgments}

We thank Sem H. Phan (Department of Pathology, University of Michigan Medical School, Ann Arbor, Michigan, USA) for the Acta2 promoter plasmid. The work was in part supported by funds from the Florida Department of Health grants $4 \mathrm{~KB} 07$ and 09BN12-23092 (to J.L.). L.W. was supported by grants from the NIH (R01DE023641 and R21CA187730).

Address correspondence to: Jianrong Lu, Department of Biochemistry and Molecular Biology, University of Florida College of Medicine, Gainesville, Florida 32610-3633, USA. Phone: 352.273.8200; E-mail:jrlu@ufl.edu.
1. Harris TJ, Tepass U. Adherens junctions: from molecules to morphogenesis. Nat Rev Mol Cell Biol. 2010;11(7):502-514.

2. Hay ED. An overview of epithelio-mesenchymal transformation. Acta Anat (Basel). 1995;154(1):8-20.

3. Kalluri R, Weinberg RA. The basics of epithelial-mesenchymal transition. J Clin Invest. 2009;119(6):1420-1428.

4. Trimboli AJ, et al. Direct evidence for epithelialmesenchymal transitions in breast cancer. Cancer Res. 2008;68(3):937-945.

5. Yu M, et al. Circulating breast tumor cells exhibit dynamic changes in epithelial and mesenchymal composition. Science. 2013;339(6119):580-584.

6 . Zhao $\mathrm{Z}$, et al. In vivo visualization and characterization of epithelial-mesenchymal transition in breast tumors. Cancer Res. 2016;76(8):2094-2104

7. Thiery JP, Acloque H, Huang RY, Nieto MA. Epithelial-mesenchymal transitions in development and disease. Cell. 2009;139(5):871-890.

8. Christofori G. New signals from the invasive front. Nature. 2006;441(7092):444-450.

9. Perl AK, Wilgenbus P, Dahl U, Semb H, Christofori $\mathrm{G}$. A causal role for E-cadherin in the transition from adenoma to carcinoma. Nature. 1998;392(6672):190-193.

10. Tsai JH, Donaher JL, Murphy DA, Chau S, Yang J. Spatiotemporal regulation of epithelialmesenchymal transition is essential for squamous cell carcinoma metastasis. Cancer Cell. 2012;22(6):725-736.

11. Chui MH. Insights into cancer metastasis from a clinicopathologic perspective: EpithelialMesenchymal Transition is not a necessary step. Int JCancer. 2013;132(7):1487-1495.

12. Bill R, Christofori G. The relevance of EMT in breast cancer metastasis: Correlation or causality? FEBS Lett. 2015;589(14):1577-1587.

13. Argast GM, et al. Inducible expression of TGF $\beta$, snail and Zeb1 recapitulates EMT in vitro and in vivo in a NSCLC model. Clin Exp Metastasis. 2011;28(7):593-614.

14. Morel AP, et al. EMT inducers catalyze malignant transformation of mammary epithelial cells and drive tumorigenesis towards claudinlow tumors in transgenic mice. PLoS Genet. 2012;8(5):e1002723.
15. Taube JH, et al. Core epithelial-to-mesenchymal transition interactome gene-expression signature is associated with claudin-low and metaplastic breast cancer subtypes. Proc Natl Acad Sci US A. 2010;107(35):15449-15454.

16. Fischer KR, et al. Epithelial-to-mesenchymal transition is not required for lung metastasis but contributes to chemoresistance. Nature. 2015;527(7579):472-476.

17. Zheng X, et al. Epithelial-to-mesenchymal transition is dispensable for metastasis but induces chemoresistance in pancreatic cancer. Nature. 2015;527(7579):525-530.

18. Whittle MC, et al. RUNX3 controls a metastatic switch in pancreatic ductal adenocarcinoma. Cell. 2015;161(6):1345-1360.

19. Peinado H, Olmeda D, Cano A. Snail, Zeb and bHLH factors in tumour progression: an alliance against the epithelial phenotype? Nat Rev Cancer 2007;7(6):415-428.

20. Lamouille S, Xu J, Derynck R. Molecular mechanisms of epithelial-mesenchymal transition. Nat Rev Mol Cell Biol. 2014;15(3):178-196.

21. Mani SA, et al. The epithelial-mesenchymal transition generates cells with properties of stem cells. Cell. 2008;133(4):704-715.

22. Miller FR, Santner SJ, Tait L, Dawson PJ. MCF10DCIS.com xenograft model of human comedo ductal carcinoma in situ. J Natl Cancer Inst. 2000;92(14):1185-1186.

23. Hristov M, Erl W, Weber PC. Endothelial progenitor cells: mobilization, differentiation, and homing. Arterioscler Thromb Vasc Biol. 2003;23(7):1185-1189.

24. Jahn SC, et al. An in vivo model of epithelial to mesenchymal transition reveals a mitogenic switch. Cancer Lett. 2012;326(2):183-190.

25 . Bergers G, Song S. The role of pericytes in bloodvessel formation and maintenance. Neuro-oncology. 2005;7(4):452-464.

26. Armulik A, Genové G, Betsholtz C. Pericytes: developmental, physiological, and pathological perspectives, problems, and promises. Dev Cell. 2011;21(2):193-215.

27. Winkler EA, Bell RD, Zlokovic BV. Central nervous system pericytes in health and disease. Nat Neurosci. 2011;14(11):1398-1405.

28. Díaz-Flores L, et al. Pericytes. Morphofunction, interactions and pathology in a quiescent and activated mesenchymal cell niche. Histol Histopathol. 2009;24(7):909-969.

29. Hosaka K, et al. Tumour PDGF-BB expression levels determine dual effects of anti-PDGF drugs on vascular remodelling and metastasis. Nat Commun. 2013;4:2129.

30. Massagué J. TGFbeta in cancer. Cell. 2008; 134(2):215-230.

31. Tang M, et al. The malignant brain tumor (MBT) domain protein SFMBT1 is an integral histone reader subunit of the LSD1 demethylase complex for chromatin association and epithelial-to-mesenchymal transition. J Biol Chem. 2013;288(38):27680-27691.

32. Ozerdem U, Grako KA, Dahlin-Huppe K, Monosov E, Stallcup WB. NG2 proteoglycan is expressed exclusively by mural cells during vascular morphogenesis. Dev Dyn. 2001;222(2):218-227.

33. Darland DC, D'Amore PA. TGF beta is required for the formation of capillary-like structures in three-dimensional cocultures of 10T1/2 and endothelial cells. Angiogenesis. 2001;4(1):11-20.

34. Bryan BA, D'Amore PA. Pericyte isolation and use in endothelial/pericyte coculture models. Meth Enzymol. 2008;443:315-331.

35. Shao R, Guo X. Human microvascular endothelial cells immortalized with human telomerase catalytic protein: a model for the study of in vitro angiogenesis. Biochem Biophys Res Commun. 2004;321(4):788-794.

36. Li F, et al. Endothelial Smad 4 maintains cerebrovascular integrity by activating $\mathrm{N}$-cadherin through cooperation with Notch. Dev Cell. 2011;20(3):291-302.

37. Gerhardt H, Wolburg H, Redies C. N-cadherin mediates pericytic-endothelial interaction during brain angiogenesis in the chicken. Dev Dyn. 2000;218(3):472-479.

38. Keogh MC, Chen D, Schmitt JF, Dennehy U, Kakkar VV, Lemoine NR. Design of a muscle cell-specific expression vector utilising human vascular smooth muscle alpha-actin regulatory elements. Gene Ther. 1999;6(4):616-628.

39. Brockschnieder D, Lappe-Siefke C, Goebbels S, Boesl MR, Nave KA, Riethmacher D. Cell depletion due to diphtheria toxin fragment $\mathrm{A}$ after Cre-mediated recombination. Mol Cell Biol. 
2004;24(17):7636-7642.

40. Ivanova A, Signore M, Caro N, Greene ND, Copp AJ, Martinez-Barbera JP. In vivo genetic ablation by Cre-mediated expression of diphtheria toxin fragment A. Genesis. 2005;43(3):129-135.

41. Hallmann R, Mayer DN, Berg EL, Broermann $\mathrm{R}$, Butcher EC. Novel mouse endothelial cell surface marker is suppressed during differentiation of the blood brain barrier. Dev Dyn . 1995;202(4):325-332.

42. Carmeliet P, Jain RK. Molecular mechanisms and clinical applications of angiogenesis. Nature. 2011;473(7347):298-307.

43. Morikawa S, Baluk P, Kaidoh T, Haskell A, Jain $\mathrm{RK}, \mathrm{McD}$ onald DM. Abnormalities in pericytes on blood vessels and endothelial sprouts in tumors. Am J Pathol. 2002;160(3):985-1000.

44. Raza A, Franklin MJ, Dudek AZ. Pericytes and vessel maturation during tumor angiogenesis and metastasis. Am J Hematol. 2010;85(8):593-598.

45. Hanahan D, Weinberg RA. Hallmarks of cancer: the next generation. Cell. 2011;144(5):646-674.

46. Dang DT, et al. Hypoxia-inducible factor-1 target genes as indicators of tumor vessel response to vascular endothelial growth factor inhibition. Cancer Res. 2008;68(6):1872-1880.

47. Cooke VG, et al. Pericyte depletion results in hypoxia-associated epithelial-to-mesenchymal transition and metastasis mediated by met signaling pathway. Cancer Cell. 2012;21(1):66-81.

48. van Beijnum JR, Nowak-Sliwinska P, Huijbers EJ, Thijssen VL, Griffioen AW. The great escape; the hallmarks of resistance to antiangiogenic therapy. Pharmacol Rev. 2015;67(2):441-461.

49. Heldin CH, Westermark B. Mechanism of action and in vivo role of platelet-derived growth factor. Physiol Rev. 1999;79(4):1283-1316.

50. Campbell CI, Moorehead RA. Mammary tumors that become independent of the type I insulinlike growth factor receptor express elevated levels of platelet-derived growth factor receptors. BMC Cancer. 2011;11:480.

51. Jechlinger M, et al. Expression profiling of epithelial plasticity in tumor progression. Oncogene. 2003;22(46):7155-7169.

52. Jechlinger M, et al. Autocrine PDGFR signaling promotes mammary cancer metastasis. J Clin Invest. 2006;116(6):1561-1570.

53. Steller EJ, et al. PDGFRB promotes liver metastasis formation of mesenchymal-like colorectal tumor cells. Neoplasia. 2013;15(2):204-217.

54. Thomson S, Petti F, Sujka-Kwok I, Epstein D, Haley JD. Kinase switching in mesenchymallike non-small cell lung cancer lines contributes to EGFR inhibitor resistance through pathway redundancy. Clin Exp Metastasis. 2008;25(8):843-854.

55. van Roy F. Beyond E-cadherin: roles of other cadherin superfamily members in cancer. Nat Rev Cancer. 2014;14(2):121-134.

56. Wheelock MJ, Shintani Y, Maeda M, Fukumoto Y, Johnson KR. Cadherin switching. J Cell Sci. 2008;121(Pt 6):727-735.

57. Lugassy C, et al. Pericytic-like angiotropism of glioma and melanoma cells. Am J Dermatopathol. 2002;24(6):473-478.

58. Olar A, Aldape KD. Using the molecular classification of glioblastoma to inform personalized treatment. J Pathol. 2014;232(2):165-177.

59. Cheng L, et al. Glioblastoma stem cells generate vascular pericytes to support vessel function and tumor growth. Cell. 2013;153(1):139-152.

60. Hendrix MJ, Seftor EA, Hess AR, Seftor RE. Vasculogenic mimicry and tumour-cell plasticity: lessons from melanoma. Nat Rev Cancer. 2003;3(6):411-421.

61. Ricci-Vitiani L, et al. Tumour vascularization via endothelial differentiation of glioblastoma stemlike cells. Nature. 2010;468(7325):824-828.

62. Wang R, et al. Glioblastoma stem-like cells give rise to tumour endothelium. Nature. 2010;468(7325):829-833.

63. Soda Y, et al. Transdifferentiation of glioblastoma cells into vascular endothelial cells. Proc Natl Acad Sci U S A. 2011;108(11):4274-4280.

64. Francescone R, et al. Glioblastoma-derived tumor cells induce vasculogenic mimicry through Flk-1 protein activation. J Biol Chem. 2012;287(29):24821-24831.

65. Dunleavey JM, et al. Vascular channels formed by subpopulations of PECAM1 ${ }^{+}$melanoma cells. Nat Commun. 2014;5:5200.

66. Eisen MB, Spellman PT, Brown PO, Botstein D. Cluster analysis and display of genome-wide expression patterns. Proc Natl Acad Sci US A. 1998;95(25):14863-14868. 